\title{
Pseudoaneurisma de la arteria carótida interna extracraneana: reporte de tres casos y revisión de la literatura
}

\section{Extracranial Internal Carotid Artery Pseudoaneurysm: Case Reports and a Review of the Literature}

Gonzalo Bustamante F. ', Walter Feuerhake M.

\begin{abstract}
Introduction. Carotid artery dissections are responsible for approximately $20 \%$ of ischemic stroke in young adults. Pseudoaneurysms are detected angiographically in 5\%-40\% of carotid artery dissections. Objectives. To present three cases of carotid artery dissection with pseudoaneurysm formation and analyze literature in terms of the natural evolution and therapeutic alternatives of the disease in order to determine the best options for treatment. Methods. Three carotid artery pseudoaneurysm cases presented during 2002 in our Department of Neurology were reviewed. Research was done using Medline and specialized Neurology and Neurosurgery articles published between 1980 and 2003, using the key words pseudoaneurysm and extracranial carotid artery dissection. Results. The clinical course of the three cases presented was benign. There was no evidence of rupture or embolization of the pseudoaneurysm during the 22 month mean follow-up period. The size of the pseudoaneurysm was reduced significantly in the first and second cases, $70 \%$ and $90 \%$, respectively. The third case had a contralateral pseudoaneurysm to the symptomatic carotid artery dissection, which remained unchanged in the angiographic controls. All cases were treated early with anticoagulants followed by antiplatelet therapy. Conclusions. Our cases had a benign outcome, as did those reported in literature. With this good prognosis, conservative management seems to be most reasonable course of action.
\end{abstract}

Key words: pseudoaneurysm, carotid artery dissection, case report.

Rev Chil Neuro-Psiquiat 2005; 43(1): 17-24

\section{Introducción}

$\mathrm{E}$ ntre la gran variedad de síndromes cerebrovasCulares, la disección de la arteria carótida, es una entidad infrecuente, especialmente el pseudoaneurisma. Muchas veces tiene una presentación oligo- sintomática, como dolor cervical o facial, síndrome de Horner, trastornos isquémicos persistentes o transitorios retinianos o cerebrales o, simplemente asintomático. Esto hace que su diagnóstico requiera de un alto índice de sospecha (1).

La evolución natural de esta entidad no ha sido

Servicio de Neurología, Clínica Santa María. Santiago, Chile (WF).
1 Interno de medicina, Facultad de Medicina Universidad de Los Andes, Santiago, Chile.
Conflicto de intereses: los autores no refieren posibles conflictos de intereses.

Recibido: septiembre 2004

Aceptado: diciembre 2004

La Revista Chilena de Neuro Psiquiatría está disponible en www.sonepsyn.cl 
estudiada a cabalidad, así como tampoco se ha determinado con certeza su manejo.

\section{Epidemiología}

Es una patología poco común. En las mayores series se ha logrado definir una incidencia de disección de la arteria carótida interna (DACI) de 2,6/100.000 habitantes de cualquier edad, con una edad promedio de presentación entre 40-46 años. No existe diferencia según sexo. La importancia está en que la disección carotídea es causa importante de accidentes cerebrovasculares en gente joven. $\mathrm{Da}$ cuenta aproximadamente de un $20 \%$ de los ACV en menores de 30 años (2).

\section{Patogénesis y fisiopatología}

La lesión inicial afecta a la íntima de la pared produciéndose un hematoma intramural. Usualmente, esta lesión comienza a unos $2 \mathrm{~cm}$ desde el bulbo carotídeo y se extiende hacia distal siguiendo la dirección del flujo. La extensión distal varía, pero generalmente se detiene a nivel de la base del cráneo, en la entrada de la arteria a través del foramen óseo. De acuerdo al tamaño del hematoma intramural, se puede ocluir el lumen de forma parcial o total. Puede ocurrir una recanalización luego de una oclusión aguda, a través del lumen fisiológico de la arteria o a través de un falso lumen, formado por la membrana disecante y la membrana muscular remanente. Cuando se comprometen las capas más superficiales de la arteria, la disección puede llevar a la formación de un pseudoaneurisma, que se diferencia de un aneurisma verdadero, en que éste contiene las tres capas de una arteria normal.

La fisiopatología de las manifestaciones clínicas incluyen la disrupción de las fibras simpáticas de la pared carotídea que llevan a configurar el síndrome de Horner completo o incompleto, ipsilateral. La irritación de la nervi vasorum, tanto por la presión intramural secundaria al hematoma intramural o la disrupción de sus componentes pueden constituir el origen del dolor del lado de la lesión. Las manifestaciones isquémicas derivan de embolismo arterio-arterial (por liberación de material su- bendotelial trombogénico) o por el compromiso hemodinámico de la perfusión cerebral, derivado del estrechamiento del lumen arterial significativo o por su oclusión completa. Sin embargo, la secuencia exacta del evento desencadenante, del crecimiento aneurismático y el comienzo de los síntomas, es todavía poco claro.

\section{Etiología}

Variadas son las causas descritas que pueden contribuir o que se relacionan con la aparición de la disección carotídea. Traumas menores de cabeza y cuello, giros bruscos del cuello o esfuerzo físico (tos, vómitos profusos, accidentes de tránsito, manipulación quiropráctica del cuello, etc.). Éstos serían capaces de comprimir la carótida interna contra los procesos transversos de las vértebras superiores, causando el daño inicial de la íntima.

Cambios estructurales pueden predisponer a la disección de arteria carótida extracraneana. La displasia fibromuscular, caracterizada por proliferación tisular fibrosa, hiperplasia muscular lisa, y destrucción fibroelástica, produce estrechamiento segmentario de arterias de tamaño mediano y evidenciable angiográficamente en un 15\% de los pacientes con DACI. Distintas colagenopatías, como el Síndrome de Marfán, el Síndrome de Ehlers-Danlos tipo IV, la deficiencia de a1-antitripsina y la mutación puntual del colágeno tipo 1 , se han asociado a la disección carotídea. El rol de la ateroesclerosis como factor predisponente para la disección carotídea aún es incierto (3).

La hipertensión arterial es el único factor de riesgo para la enfermedad cerebrovascular, que se encuentra con mayor frecuencia en pacientes con disección carotídea en comparación con la población general (4).

\section{Casos clínicos}

\section{Caso 1}

Paciente de 26 años, sana, con historia de jaquecas, que inicia cefalea holocránea persistente de predominio hemicránea izquierda, luego de haber presentado cuadro gripal que se manejó 

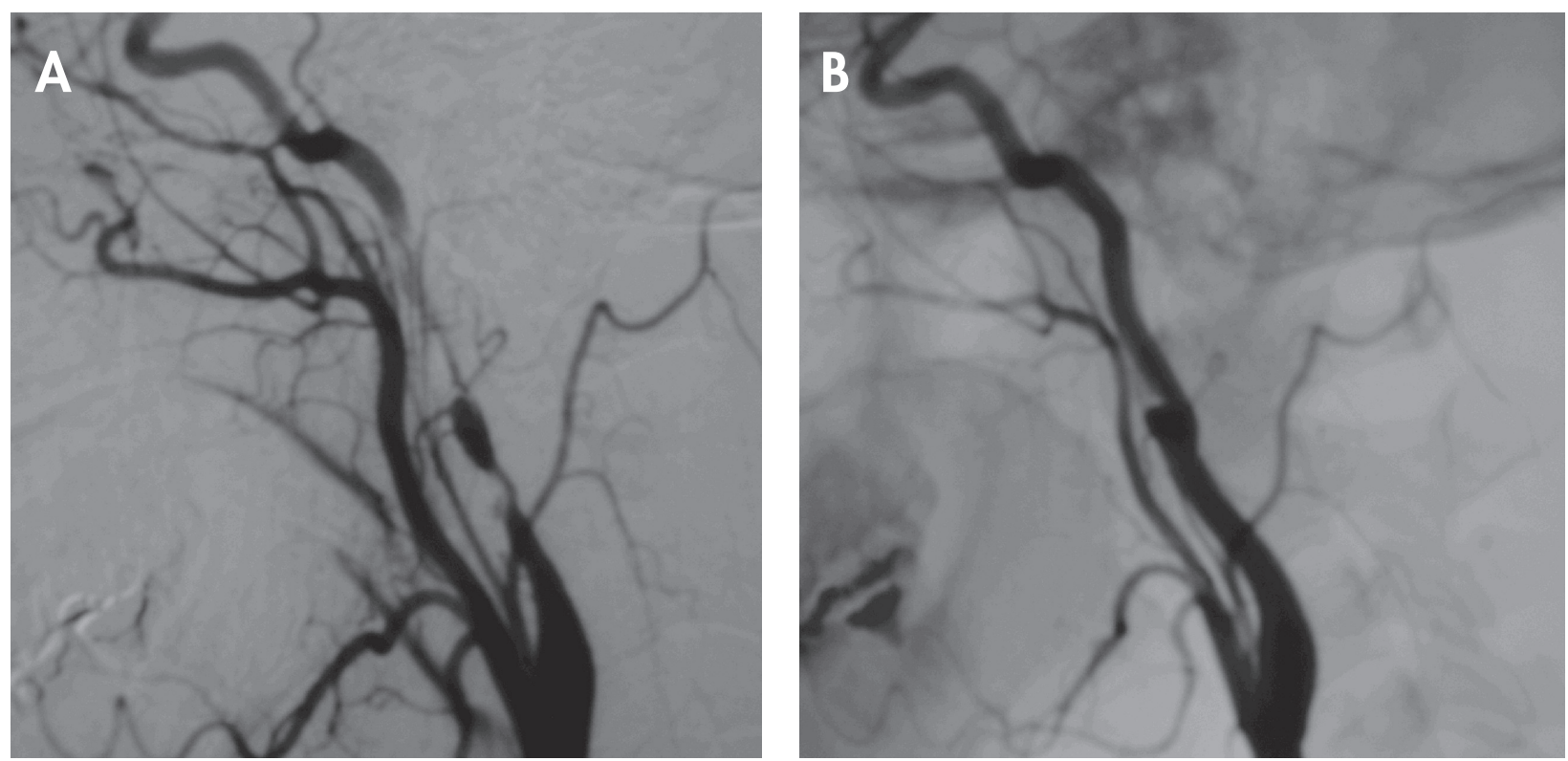

Figura 1. Angiografía cervical con sustracción digital. A. Disección de la porción cervical de la arteria carótida interna izquierda con pseudoaneurisma y estenosis filiforme secundaria. B. Control angiográfico a las 12 semanas de evolución, se observa una disminución en el tamaño del pseudoaneurisma y un flujo adecuado de la ACl.

sintomáticamente con un antigripal. Persiste con cefalea y presenta hemiparesia de predominio braquial derecha transitoria, de 15 horas de duración. A su ingreso el examen neurológico es normal. La Resonancia Magnética (RNM) por difusión muestra un infarto del territorio de la arteria cerebral media izquierda. La angiografía cerebral mostró disección de la arteria carótida interna izquierda con pseudoaneurisma y estenosis filiforme secundaria (Figura 1A). Se inicia anticoagulación y analgesia. Evoluciona sin focalidad neurológica y se da de alta en buenas condiciones generales. Permanece asintomática y se realiza control angiográfico a las 8 semanas que muestra el pseudoaneurisma con resolución de la estenosis. Controles clínicos posteriores sin evidencia de focalidad neurológica. Se realiza control a las 12 semanas que muestra una disminución del tamaño del pseudoaneurisma en un $70 \%$ y un flujo adecuado en arteria carótida interna (Figura 1B). Se mantiene anticoagulada por 3 meses, continuando con aspirina.

\section{Caso 2}

Paciente de 44 años con antecedentes de dislipidemia, tabaquismo y sobrepeso. Estando en buenas condiciones generales presenta, al vestirse, cervicalgia intensa de aproximadamente 15 minutos de duración que permanece durante todo el día, pero con una intensidad menor. Al quinto día presenta un síncope durante la ducha, refiriendo luego una leve paresia braquial derecha de una hora de duración. Ese mismo día consulta en un servicio de urgencia, donde se constata un examen neurológico normal. Es enviada a su domicilio e incluso se realiza exámenes ambulatorios, incluido un Test de esfuerzo. Al día siguiente, al levantarse presenta mareos e instalación brusca de una hemiparesia faciobraquial derecha asociado a disartria.

$\mathrm{Al}$ ingreso sólo se constata una discreta paresia braquial derecha. La RNM por difusión muestra una lesión hiperintensa en la cápsula anterior izquierda. La angiografía muestra una disección de la arteria carótida interna izquierda con formación de un pseudoaneurisma (Figura 2A) Se inicia anticoagulación sistémica. Evoluciona favorablemente, asintomática y sin déficit neurológico. Recibe anticoagulación por 6 meses y luego se mantiene con aspirina. El control imagenológico a los 8 meses muestra regresión casi completa del pseudoaneurisma (Figura 2B). 

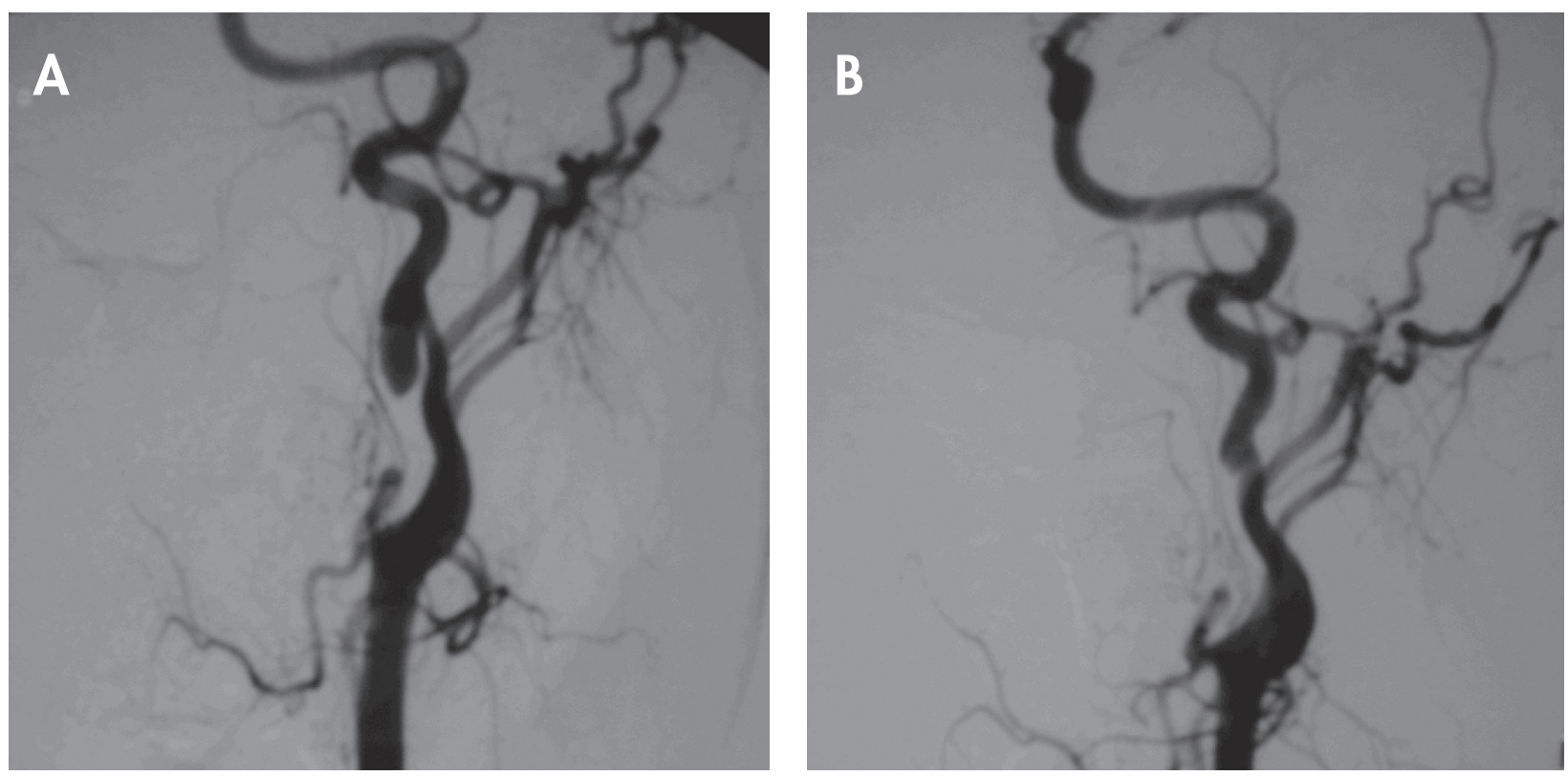

Figura 2. Angiografía cervical con sustracción digital. A. Disección del segmento cervical de ACl izquierda, con formación de pseudoaneurisma. B. Control angiográfico a los 8 meses, con resolución casi completa del pseudoaneurisma.

\section{Caso 3}

Paciente sano de 43 años con antecedentes de cuadro depresivo en tratamiento con Clonazepam y Fluoxetina. Luego de viaje en avión de 30 horas de duración presenta dolor cervical persistente que no responde a analgésicos comunes por un período de 24 horas, iniciando posteriormente un cuadro de déficit motor del hemicuerpo derecho y afasia mixta, por lo que consulta al servicio de urgencia.

$\mathrm{Al}$ ingreso se constata discreta hemiparesia braquiocrural derecha y afasia. La RMN por difusión muestra infarto en territorio de ACM izquierda. La angiografía cervical mostró disección completa con oclusión de la arteria carótida izquierda (Figura 3A). Además, un pseudoaneurisma en arteria carótida interna derecha, en el segmento subpetroso (Figura 3B). Este último sin manifestaciones clínicas. Evoluciona estable, con regresión parcial de los elementos focales. Se da de alta en buenas condiciones generales, deambulando, con afasia mixta. Permanece anticoagulado por 6 meses, para luego continuar con aspirina. Controles clínicos posteriores muestran recuperación en el déficit motor y permanencia de la afasia. Controles posteriores no muestran cambios en el pseudoaneurisma carotídeo derecho.

\section{Discusión}

Existen buenos reportes de la presentación clínica de la disección de la arteria carótida interna. Los síntomas más comunes de la DACI son la cefalea y/o dolor cervical. El dolor característico es de inicio súbito, severo, ipsilateral en la región periorbitaria, periauricular o cervical alto, pero existe una amplia variación. La cefalea puede ser moderada, difusa o bilateral. Es el síntoma que se presenta con más frecuencia en las diferentes series publicadas, desde un 60 a 92\% (5). La asociación con el síndrome de Horner es característica, ya sea en su forma incompleta o su forma clásica. Se ve en hasta un $50 \%$ de los DACI (1).

Los eventos isquémicos (Infarto/TIA) en los casos de DACI aparecen en la mayoría de los casos dentro de los primeros 30 días. En una serie de 80 pacientes con disección carotídea extracraneana, el $88 \%$ de los pacientes que hicieron isquemia, fue dentro de la primera semana (5).

Un estudio suizo reciente (6) de seguimiento a 6 años de DACI unilateral, bajo tratamiento médico (antiagregantes), mostró una tasa anual de 0,7\% para eventos isquémicos del territorio carotídeo 

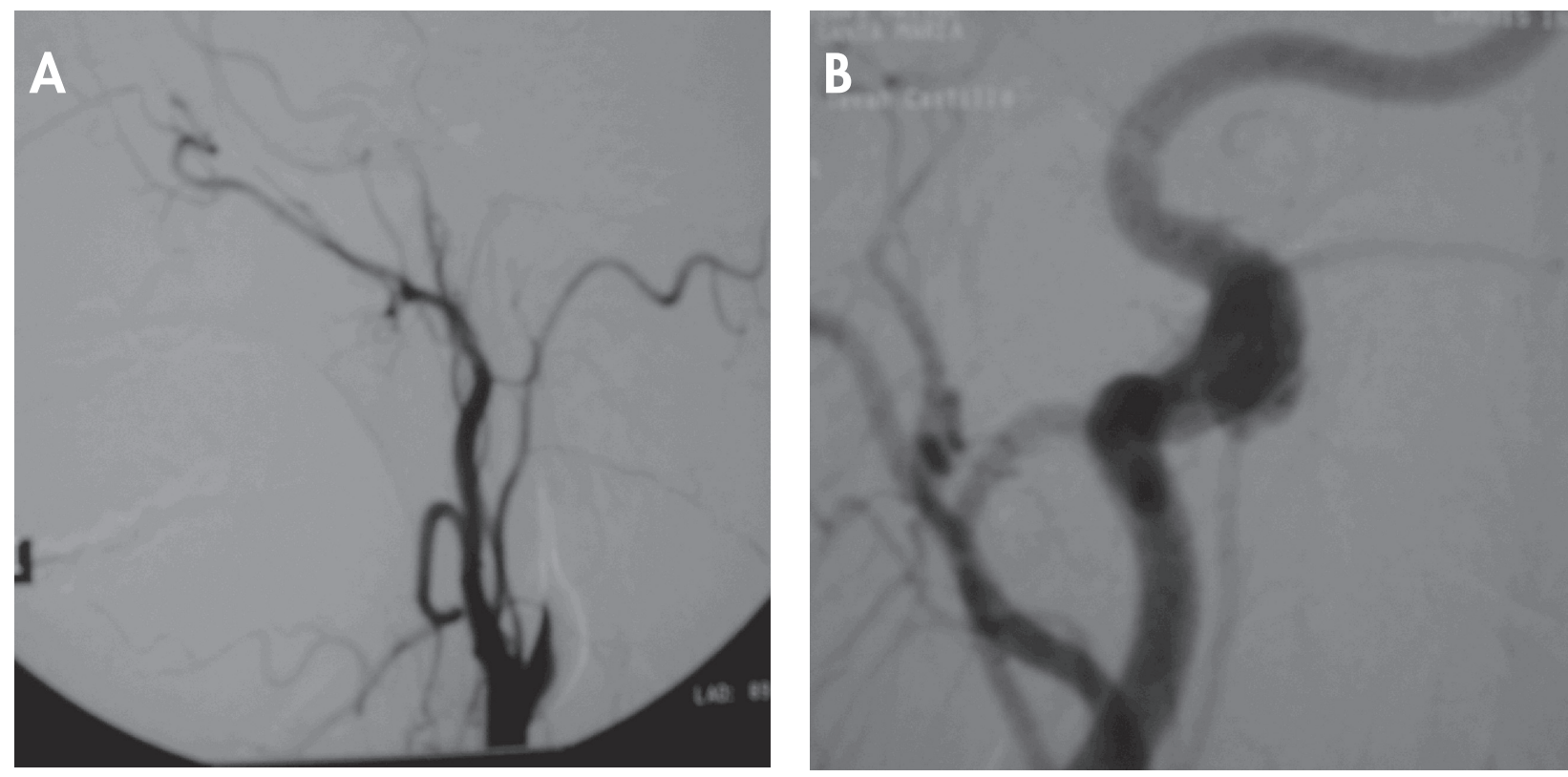

Figura 3. Angiografía cervical con sustracción digital. A. Disección ACl izquierda con oclusión completa. B. Pseudoaneurisma de la porción petrosa de $\mathrm{ACl}$ derecha, hallazgo asintomático.

ipsilateral y un $1,4 \%$ para cualquier AVE y tasas equivalentes a la mitad para eventos isquémicos transitorios. Más aún, los eventos isquémicos no se relacionaron con la persistencia de estenosis u oclusión severa de la carótida.

Durante la fase inicial de la DACI, puede formarse el pseudoaneurisma, el cual en pocas semanas puede crecer. Con el tiempo pueden permanecer sin cambios, disminuir de tamaño o resolverse (7). La resolución de los aneurismas es más común en las disecciones espontáneas que en las traumáticas. En la etapa más crónica, los pseudoanurismas raramente crecen o se rompen. Sin embargo, pueden convertirse en un nido de embolización distal. Esto probablemente sea más frecuente en los traumáticos que en los espontáneos (8).

Se encontraron sólo dos estudios de seguimiento a largo plazo de los aneurismas desarrollados luego de una DACI. En el estudio de Guillon et al (9) se encontró que un 27,5\% de los pacientes estudiados por DACI durante 36,9 meses desarrollaron aneurismas. La evolución clínica de estos pacientes fue excelente, no hubo recurrencias, ni eventos isquémicos, tampoco signos neurológicos de compresión. La evolución radiológica también fue favorable: un $65 \%$ se mantuvo sin cambios, un caso (5\%) se resolvió a los 6 meses, y un 30\% disminuyó de tamaño. La estenosis arterial se resolvió en $72 \%$ de los aneurismas estenóticos y persistió en un 25\%. En otro estudio similar, de Touzé et al (10), tampoco hubo eventos isquémicos recurrentes, ruptura, ni signos compresivos locales, luego de un seguimiento a 3 años. Así como en el estudio de Guillon, tampoco se observó aumento en el tamaño de los aneurismas durante el seguimiento radiológico, además un $18 \%$ disminuyó de tamaño y un $46 \%$ se mantuvo sin cambios. La persistencia de los aneurismas, fue de un $95 \%$ y $67 \%$ para ambos estudios, respectivamente. En ambos estudios, los pacientes fueron manejados de forma conservadora, básicamente con anticoagulación inicial y luego uso de aspirina en dosis antiagregante.

En nuestros casos, la evolución también fue favorable. Todos recibieron anticoagulación inicial entre 3 a 6 meses, para luego continuar con aspirina. Los dos primeros casos no presentaron secuelas neurológicas y el tercer paciente tuvo mejoría motora con persistencia de una afasia mixta. Este último fue el que presentó una disección completa de la arteria carótida izquierda, presentando además un 
pseudoaneurisma carotídeo contralateral asintomático. En los dos primeros casos hubo reducción de tamaño del pseudoaneurisma y el tercero se mantuvo sin cambios. Ninguno de los tres pacientes presentó eventos isquémicos, signos compresivos o de crecimiento del pseudoaneurisma, a los 18 meses de seguimiento.

Se han descrito diversas técnicas quirúrgicas para la resolución de los pseudoaneurismas carotídeos extracraneanos secundarios a disección. Básicamente existen tres técnicas: la ligadura de arteria carótida, resección del aneurisma con reconstrucción carotídea y el bypass carotídeo cervical-intracraneal. Las series son pequeñas y la mayoría de los pacientes operados corresponden a aneurismas por disección traumática o iatrogénica y aneurismas ateroescleróticos de la arteria carótida. El registro más grande publicado es de sólo 67 pacientes operados entre 1960 y 1995 , con una tasa de complicaciones global de un $10 \%$ para mortalidad y de un $6 \%$ para lesión de nervios craneales (11).

El tratamiento quirúrgico se ha reservado para aquellos aneurismas residuales sintomáticos, la mayoría de ellos por disección traumática de la arteria carótida interna extracraneana (12).

Por otro lado, también se ha reportado el manejo de los pseudoaneurismas mediante técnicas percutáneas, principalmente aquellos pseudo-aneurismas post traumáticos y/o iatrogénicos.

Liu AY, et al (13) ha publicado la mayor serie de disección carotídea tratadas con stents $(n=7)$, debido a persistencia de la disección, formación de pseudoaneurisma, o avance de la sintomatología, a pesar de la terapia médica. De los pacientes tratados por pseudoaneurisma $(n=4)$, dos presentaron oclusión espontánea del aneurisma al momento del procedimiento, un tercero a los 18 meses de instalado el stent y el cuarto presentó una disminución del tamaño en el seguimiento posterior. Todos los casos permanecieron asintomáticos durante el período de seguimiento (10-67 meses). Estos autores sugieren la instalación de un stent en caso de estenosis inducida por la disección que no responde a terapia médica, síntomas isquémicos o tromboembólicos, disección en un paciente con contraindicación de anticoagulación y disección iatrogénica con estenosis preoclusiva severa.

Sin embargo, dado la escasa experiencia que existe con este tipo de procedimiento y la buena evolución de la disección y el pseudoaneurisma con tratamiento conservador, hace que la terapia endovascular no sea considerada como tratamiento de primera línea, al igual que el abordaje quirúrgico.

\section{Conclusión}

Nuestros casos clínicos, a pesar del escaso número, tuvieron un comportamiento benigno, al igual que lo reportado en la literatura. No existen estudios prospectivos randomizados controlados en relación al manejo de las formas aneurismáticas, de manera que la estrategia adecuada para la prevención secundaria de estos pacientes aún no está definida. Pero, la evidencia actual apunta que, al igual que la disección carotídea espontánea, las formas aneurismáticas son de buen pronóstico y tienen un comportamiento benigno en el tiempo. De manera que, un manejo conservador que incluya anticoagulación inicial, con el objetivo de prevenir embolización cerebral, y luego hacer una prevención secundaria con antiagregantes plaquetarios, parece razonable.

Agradecimientos:

Dr. José Tevah C. Por su colaboración con el material fotográfico (imágenes).

E.U. Soledad Urrutia U. Por la ayuda brindada en la búsqueda de material de registro clínico. 
Introducción. La disección de arteria carótida da cuenta de aproximadamente un 20\% de los accidentes cerebrovasculares (ACV) isquémicos de pacientes jóvenes. En este grupo se detectan angiográficamente entre un 5 y 40\% de formación de pseudoaneurismas. Objetivos. Presentar tres casos de disección espontánea de la arteria carótida interna extracraneana con formación de pseudoaneurisma y analizar la literatura en relación a la evolución natural de la enfermedad y las alternativas terapéuticas descritas, para definir la conducta terapéutica más adecuada. Métodos. Se revisan 3 casos de pseudoaneurisma carotídeo observados durante 2002 en el servicio de neurología de nuestra institución. Utilizando las palabras claves: pseudoaneurisma, disección de arteria carótida interna extracranana, se hizo una búsqueda en Medline, revistas especializadas de neurología y neurocirugía entre los años 1980 y 2004. Resultados. Los tres casos presentados tuvieron un comportamiento benigno en el tiempo. No hubo evidencia de ruptura o embolización de los pseudoaneurismas durante los 22 meses de seguimiento. Los dos primeros casos presentaron una reducción significativa del tamaño del pseudoaneurisma, un 70 y $90 \%$, respectivamente. El tercer caso presentó un pseudoaneurisma contralateral a disección carotídea sintomática, que se mantuvo sin cambios en los controles angiográficos. Todos los casos fueron manejados con anticoagulación inicial y luego con aspirina. Conclusiones. Nuestros casos clínicos tuvieron un comportamiento benigno, al igual que lo reportado en la literatura. Dado el buen pronóstico de esta patología, un manejo conservador parece lo más razonable.

Palabras claves: pseudoaneaneurisma, disección de arteria carótida, reporte de casos.

\section{Referencias}

1. Stapf C, Elkind MS, Mohr JP. Carotid artery dissection. Annu Rev Med 2000; 51: 329-347

2. Bogousslavsky J, Regli F. Isquemic stroke in adults younger than 30 years of age. Cause and prognosis. Arch Neurol 1987; 44: 479-482

3. Guillon B, Lévy C, Bousser MG. Internal carotid dissection: an update. J Neurol Sci 1998; 153: 146-158

4. Ast G, Woimant F, Georges B, Laurian C, Haguenau M. Spontaneous dissection of the internal carotid artery in 68 patients. European Journal of Medicine 1993; 2: 466-472

5. Biousse V, D'Anglejan-Chatillon J, Touboul PJ, Amarenco P, Bousser MG. Time course of symptoms in extracranial carotid artery dissections. a series of 80 patients. Stroke 1995; 26 : 235-239
6. Kremer C, Mosso M, Georgiadis D, Stöckli E, Benninger D, Arnold M, et al. Carotid dissection with permanent and transient occlusion or severe stenosis; long-term outcome. Neurology 2003; 60: 271-275

7. Schievink WI, Piepgras DG, McCaffrey TV, Mokri M. Surgical treatment of extracranial internal carotid artery dissecting aneurysm. Neurosurgery 1994; 35: 809-816

8. Mokri B. Traumatic and spontaneous extracranial internal carotid artery dissections. J Neurol 1990; 237: 356-361

9. Guillon B, Brunereau L, Biusse V, Djouhri H, Lévy C, Bousser MG. Long-term follow-up of aneurysms developed during extracranial internal carotid artery dissection. Neurology 1999; 53: 117-122

10. Touzé E, Randoux B, Méary E, Arquizan C, Meder J-F, Mas J-L. Aneurysmal forms of cer- 
vical artery dissection: associated factors and outcome. Stroke 2001; 32: 418-423

11. El-Sabrout R, Cooley DA. Extracranial carotid artery aneurysms: Texas Heart Institute expirience. J Vasc Surg 2000; 31: 702-712

12. Patel JV, Rossbach MM, Cleveland TJ, Gaines PA, Beard JD. Endovascular stent-graft repair of traumatic carotid artery pseudoaneurysm. Clin Radiol 2002; 57: 308-311

13. Liu AY, Paulsen RD, Marcellus ML, Steinberg GK, Marks MP. Long-term outcomes after carotid stent placement for treatment of carotid artery dissection. Neurosurgery 1999; 45: 13681374

Correspondencia

Gonzalo Bustamante F.

Av. Simón Bolívar 8299 Casa B.

La Reina, Santiago, Chile.

Fonos: 2732469 - 09/8221138

E-mail: gbf@uandes.cl 